\title{
Importances zootechniques et parasites internes des porcs de race locale (Sus crofa domesticus) élevés au Bénin: synthèse bibliographique
}

\author{
Akouavi Carine Chimène ADOHO ${ }^{1,2,3}$, Pascal Abiodoun OLOUNLADE ${ }^{1,2,3^{*}}$, \\ Erick Virgile Bertrand AZANDO ${ }^{2,3,4}$, Sylvie HOUNZANGBE-ADOTE ${ }^{2}$ et \\ Armand Bienvenu GBANGBOCHE ${ }^{1,3}$
}

\begin{abstract}
${ }^{1}$ Unité de Recherche en Zootechnie et Système d'Elevage (URZoSE), Laboratoire des Sciences Animales et Halieutiques (LaSAH), Ecole Doctorale des Sciences Agronomiques et de l'Eau, Université Nationale d'Agriculture (EDSAE/UNA), 01 BP 55, Porto-Novo, Bénin.

${ }^{2}$ Laboratoire d'Ethnopharmacologie et de Santé Animale (LESA), Faculté des Sciences Agronomiques (FSA), Université d'Abomey Calavi (UAC), 01 BP 526 Cotonou, Bénin.

${ }^{3}$ Laboratoire de Biotechnologie et d'Amélioration Animale, Faculté des Sciences Agronomiques, Institut des Sciences Biomédicales Appliquées (ISBA), Université d'Abomey-Calavi, 01 BP 526 Cotonou, Bénin.

${ }^{4}$ Laboratoire de Recherche sur les Interactions Vecteurs, Hôtes, Agents Pathogènes (LaRIVHAP), Département des Sciences et Techniques de Production Animale et Halieutique, Faculté d'Agronomie (FA), Université de Parakou, BP 123, Parakou, Bénin. *Auteur correspondant ; Cell : +22997085468 ; E-mail: abiodouno@yahoo.fr
\end{abstract}

\begin{tabular}{ccc}
\hline Received: 08-01-2021 & Accepted: 20-08-2021 & Published: 31-08-2021 \\
\hline
\end{tabular}

\section{RESUME}

En Afrique et particulièrement au Bénin, l'élevage du porc constitue une activité génératrice de revenu. Toutefois, les acteurs de ce secteur sont confrontés à de nombreuses difficultés dont le parasitisme qui entraîne une baisse de production et de productivité. Le présent travail a pour but de faire une revue de littérature sur les parasites qui affectent l'élevage des porcs en Afrique et particulièrement au Bénin. De l'analyse des données collectées, il ressort que trois types d'élevage sont pratiqués au Bénin : l'élevage traditionnel, prédominant et pratiqué à $65 \%$, l'élevage semi-intensif ou semi moderne à $32 \%$ et enfin l'élevage intensif ou moderne à $3 \%$. L'amélioration des conditions d'élevage et particulièrement l'alimentation du porc indigène d'Afrique est favorable à l'expression de son potentiel zootechnique. Malgré l'optimalisation des rations, les performances zootechniques du porc local restent encore faibles par rapport à celles des races améliorées. Très peu d'études ont abordé la prévalence des parasites internes du porc au plan national, mais parmi les parasites rapportés, on a noté : Ascaris suum, Strongyloides ransomi, Trichuris suis, le genre Hyostrongylus et d'autres parasites internes du porc. Il s'impose de mener une étude sur les parasites du porc au Bénin afin de déterminer la prévalence des différents parasites et le danger que ces derniers représentent pour le développement de cette filière.

(C) 2021 International Formulae Group. All rights reserved.

Mots clés : Elevage du porc local, Parasites du porc, Prévalence, Zootechnie, Sécurité sanitaire, Bénin. 


\title{
Zootechnical importance and internal parasites of local pigs (Sus crofa domesticus) raised in Benin: bibliographic synthesis
}

\begin{abstract}
In Africa, and particularly in Benin, pig farming is an income-generating activity. However, the actors of this sector are confronted with many difficulties, including parasitism which leads to a decrease in production and productivity. The aim of this study is to review the literature on parasites affecting pig farming in Africa, particularly in Benin. From the analysis of the data collected, it appears that three types of livestock production are practised in Benin: traditional livestock production, which is predominant and practised at $65 \%$, semiintensive or semi-modern livestock production at $32 \%$ and finally intensive or modern livestock production at $3 \%$. The improvement of breeding conditions and particularly the feeding of the indigenous African pig is favorable to the expression of its zootechnical potential. Despite the optimization of rations, the zootechnical performance of local pigs is still low compared to that of improved breeds. Very few studies have addressed the prevalence of internal parasites of pigs at the national level, but among the parasites reported were: Ascaris suum, Strongyloides ransomi, Trichuris suis, the genus Hyostrongylus and other internal parasites of pigs. It is necessary to conduct a study on pig parasites in Benin in order to determine the prevalence of the different parasites and the danger that they represent for the development of this sector.
\end{abstract}

(C) 2021 International Formulae Group. All rights reserved.

Keywords: Local pig breeding, Pig parasites, Prevalence, Zootechnics, Health safety, Benin.

\section{INTRODUCTION}

La réduction de la pauvreté dans les pays en voie de développement notamment en Afrique et particulièrement au Bénin ne peut se réaliser sans la prise en compte des activités génératrices de revenus (Agbokounou et al., 2016). Parmi ces activités, l'élevage occupe une place prioritaire (Rouillé et al., 2014), tout particulièrement l'élevage du porc. Dans les campagnes notamment les villages, de nombreuses familles possèdent quelques porcs qu'elles élèvent en liberté autour de leur domicile et qu'elles utilisent de temps à autres pour la consommation en famille (Rouillé et al., 2014) mais également pour la vente.

Au Bénin, le cheptel porcin était estimé à 414000 têtes, selon les données rapportées par la Direction de l'élevage en 2013 (Direction de l'élevage, 2015) puis à 504000 têtes en 2018 (FAOSTAT-FAO, 2020). Malgré cela, le secteur est confronté à des problèmes d'ordre sanitaire notamment les parasitoses (Agbokounou et al., 2016). Même si ces dernières ne provoquent pas souvent de mortalité directe, elles sont d'une conséquence économique importante en raison des baisses de production et de productivité qu'elles engendrent (Agbokounou et al., 2016) et de la diminution de la résistance organique de l'animal parasité, ayant pour origine, la potentialisation de l'expression clinique de certaines maladies infectieuses associées (Corrégé et al., 2008). La connaissance des agents parasitaires du porc est donc importante pour optimiser la productivité du secteur, elle permet de respecter les mesures d'hygiène et de prendre aussi des mesures sanitaires supplémentaires afin de mieux préserver la santé des animaux.

Une lutte efficace et rationnelle contre les maladies parasitaires doit nécessairement passer par la connaissance parfaite des agents responsables C'est dans ce contexte que cette revue de littérature a été initiée avec pour objectif de présenter l'élevage porcin au Bénin et la prévalence des maladies parasitaires qui entravent le développement de cette filière. Elle prendra en compte la caractérisation des systèmes d'élevage porcin, les contraintes majeures de l'élevage du porc au Bénin, et les précautions à prendre pour améliorer la santé des porcs. 


\section{CARACTERISTIQUES DE L'ELEVAGE PORCIN EN AFRIQUE}

\section{Système d'élevage}

En Afrique et particulièrement au Bénin, la production du porc local est basée essentiellement sur un système d'élevage traditionnel et familial. Les caractéristiques de ce système d'élevage se rencontrent parfois en zone tropicale de l'Asie et de l'Amérique (Nonfon, 2005). Il est souvent de type villageois et de forme extensive. Ce système se retrouve dans les zones urbaines et périurbaines où sont élevées la race locale accompagnée d'autres races exogènes (Large White, Landrace, Duroc, Piétrain, Hampshire) ou les hybrides de leur croisement avec la race locale ou des produits issus de plusieurs croisements incontrôlés (Lombe et al., 2015; Lukusa, 2005). Dans le cas de l'élevage des races améliorées et de leurs croisés, les éleveurs apportent une amélioration à la forme d'élevage.

Au Bénin, l'élevage du Porc est surtout l'apanage des populations du Sud-Bénin et du centre, dans une proportion de près de $90 \%$. Ceci est surtout dû aux interdits religieux qui frappent la population du Nord Bénin, en majorité musulmane. Par ailleurs, les conditions climatiques sont peu favorables dans le Nord (une saison de pluie), alors que le Sud et le Centre bénéficient d'un climat subéquatorial à régime pluviométrique bimodal avec une amplitude thermique faible et une végétation herbacée dominée par les herbes grasses très utilisées en alimentation porcine (Ayssiwede, 2004)

Dans ce système traditionnel, l'élevage ou la conduite des troupeaux repose sur deux manières : la divagation et la claustration. Même si la divagation est généralement la plus observée en élevage traditionnel et surtout dans le milieu villageois, ces deux modes sont souvent règlementés par le calendrier des activités agricoles chez les paysans (Lombe et al., 2015) Ils sont alors saisonniers avec une claustration pendant la saison des cultures et une divagation diurne pendant la saison sèche (Mopate et al., 2010; Umutoni, 2012; Youssao et al., 2008) La majorité des éleveurs sont contraints de pratiquer la claustration permanente ou saisonnière à cause des dégâts causés par la divagation et des mesures prises à son encontre que sont les recommandations et répressions administratives sanitaires, la difficulté de maîtrise de l'animal, les conflits entre éleveurs et agriculteurs, l'impact des maladies sur la santé publique et la propagation rapide des maladies (Agbokounou, 2001; Youssao et al., 2008). Ce fait s'observe surtout dans les milieux urbains et périurbains. Les animaux disposent d'un habitat, même ceux élevés en divagation auxquels l'habitat sert de lieu de couchette et d'apport de compléments alimentaires éventuels (Agbokounou, 2001a) En claustration, les animaux sont en enclos ou attachés (FAO, 2012) A travers ces modes de conduite, l'éleveur contribue d'une manière ou d'une autre à la gestion d'un habitat, de l'alimentation, de la reproduction et parfois de celle de la santé. Ces pratiques sont généralement similaires dans la plupart des pays du continent avec quelques particularités au niveau des matériaux de construction, des habitats et des soins sanitaires prodigués aux animaux.

Notons qu'en Afrique de l'Ouest, Centrale, Australe et même à Madagascar, les habitats du porc local sont caractéristiques du type d'élevage traditionnel (Robineau, 2018). En dehors de quelques élevages disposant d'habitats en matériaux durables, la plupart des habitats du porc local de ces régions sont en matériaux locaux. Pour les habitats durables, les enclos sont des murs en brique de ciment. Compartimentés ou non, les enclos en matériaux locaux sont en banco, en bois, en bambou, en tiges de roseaux ou palmes tressées (Abdallah-Nguertoum, 1997; Agbokounou, 2001b; FAO, 2012; Randriamahefa, 2002) sans toit généralement; le sol en terre battu, nu, devenant un bourbier pendant la saison des pluies (Agbokounou, 2001b; FAO, 2012).

Aussi, soulignons l'absence des mangeoires dans les porcheries de certains pays comme le Centre Afrique (AbdallahNguertoum, 1997); et dans l'Afrique en général, les mangeoires-abreuvoirs faits d'ustensiles de fortunes (Abdallah-Nguertoum, 1997; Mopate et al., 2010 ; Nonfon, 2005); ou en bois taillé comme au Bénin, Au 
Madagascar, en Centre afrique (Mopate et al., 2010; Nonfon, 2005; Randriamahefa, 2002) Ces habitats ne respectent aucune norme zootechnique et les animaux y sont sans aucune règle d'hygiène, sans distinction de sexe ou de stade physiologique (Agbokounou, 2001a; Randriamahefa, 2002; Youssao et al., 2008).

$\mathrm{Au}$ plan sanitaire, aucun soin n'est prodigué aux animaux par les éleveurs. Le recours aux soins vétérinaires est généralement caractéristiques des zones urbaines (Missohou et al., 2001; Youssao et al., 2008). L'utilisation des plantes médicinales est courant en milieu rural ou autres produits à portée thérapeutiques pour traiter les animaux (Missohou et al., 2001).

Les pathologies observées dans ces élevages traditionnels sont dues à l'état du sol et la quasi inexistence de soins préventifs. la plus fréquente est le parasitisme (AbdallahNguertoum, 1997; Carter et al., 2013); accentué par la divagation où la propagation des maladies est rapide et facile. Aussi le polyparasitisme est caractéristique de ce système d'élevage (Nonfon, 2005).

\section{Races de porcs élevés au Bénin}

Plusieurs races de porc sont élevées au Bénin. Au Sud-Bénin, on distingue les races locales, large-white, landrace et les croisés (Djimenou et al., 2017). Une enquête réalisée au Sud-Bénin a montré que certains éleveurs gardent deux à trois races de porcs différentes au sein de leur troupeau afin d'obtenir des produits croisés (Djimenou et al., 2017). Les porcs locaux en 1994 représentaient 93,5\% du cheptel porcin au Sud du Bénin (Nonfon et al., 1994). Mais, entre 1994 et 2015, il a été noté une forte réduction de l'élevage du porc local au profit de l'élevage des croisés et des races exotiques (Djimenou et al., 2017). Selon une enquête réalisée au Sud-Bénin en 2020, Les porcs de races améliorées sont priorisés $(94,29 \%)$ (Ohouko et al., 2020).

\section{Potentiel zootechnique}

D'après Agbokounou et al., (2016), le porc local d'Afrique est caractérisé par une capacité de réaction positive aux actions d'amélioration de son élevage ou de son intensification. (Keambou et al., 2010; Koutinhouin et al., 2009; Youssao et al., 2008). Du point de vue reproduction, il est caractérisé par une précocité sexuelle élevée non exprimée dans les conditions d'élevage traditionnel. En divagation, les âges moyens au premier oestrus de 210 à 250 jours et parfois à 302 jours avec les races locales d'Afrique de l'Ouest, de l'Est et des savanes d'Afrique centrale (d'Orgeval, 1997; Mopate et al., 2011; Mushandu et al., 2005). La durée moyenne de gestation observée avec la race locale de petit format de l'Afrique de l'Ouest est de 111-114 jours et 114,6 jours pour les races exotiques (Nonfon, 2005). Les taux de mortinatalité, de mortalité naissance-sevrage et les gains moyens quotidiens des 4 premières semaines de vie et de naissance-sevrage respectivement de $5 \%$, $29 \%, 55 \mathrm{~g} / \mathrm{j}$ et $49 \mathrm{~g} / \mathrm{j}$ ont été obtenus dans le mode traditionnel amélioré contre respectivement $12 \%, 32 \%, 34 \mathrm{~g} / \mathrm{j}$ et $39 \mathrm{~g} / \mathrm{j}$ en mode traditionnel pur (Koutinhouin et al., 2009) L'élevage du porc local en Afrique de l'Ouest en conditions améliorées permet d'augmenter la productivité numérique de $51 \%$ par rapport au système d'élevage traditionnel extensif (Nonfon, 2005). La truie locale d'Afrique a une productivité pondérale ramenée à son poids, exprimée par $10 \mathrm{~kg}$ de poids vif largement supérieure à celle des truies de race améliorée (12,3 à 16,1 contre 4,1 à 8,6) (d'Orgeval, 1997).

Les performances de croissance du porc local dépendent de son mode de conduite, car par la collecte de nourriture dans la nature, l'animal ne couvre que ses seuls besoins de déplacement. En claustration ou en divagation, le poids est élevé chez les mâles castrés que les femelles $(15,5 \mathrm{~kg}$ à 360 jours contre $12,0 \mathrm{~kg})$ en milieu traditionnel villageois ; et supérieurs à celui des mâles entiers $(4,50-5,50 \mathrm{~kg}$ entre 120 et 360 jours) (Nonfon, 2005). Aussi, les porcs locaux de N'Djamena ont présenté des gains moyens quotidiens entre 0 et 6 mois de 87 et $91 \mathrm{~g} / \mathrm{j}$ respectivement chez les mâles et les femelles (Mopate et al., 2011). Notons également l'influence des saisons sur les performances de croissance du porc. En effet, au Kenya, les gains moyens quotidiens obtenus dans la zone peri urbaine ont été meilleurs (150 
$\mathrm{g} /$ jour) que ceux obtenus en zone rurale (110 g/jour) (Carter et al., 2013). Les poids obtenus en élevage traditionnel par certains auteurs sont de $31 \mathrm{~kg}$ à 8 mois à Bangui (AbdallahNguertoum, 1997), 30 à $35 \mathrm{~kg}$ à 12 mois en RDC (FAO, 2012) et $45 \mathrm{~kg}$ à 12 mois d'âge avec un poids à la naissance de $0,5 \mathrm{~kg}$ pour le porc nigérian (Anugwa \& Okwori, 2008). Ces performances du porc nigérian se rapprochent de celles de la race Muong Lay du Vietnam dont le poids à la naissance est de $0,5 \mathrm{~kg}$ avec des poids moyens à un an de $44 \mathrm{~kg}$ et $52 \mathrm{~kg}$ respectivement pour les mâles et les femelles (Do Duc, 2013). Les différents gains moyens quotidiens observés sont 46,58 et $88 \mathrm{~g} / \mathrm{j}$ (Nonfon, 2005) puis de $54 \mathrm{~g} / \mathrm{j}$ (Youssao et al., 2004) dans les élevages traditionnels du Bénin, de $88 \mathrm{~g} / \mathrm{j}$ pour les porcs du Burkina Faso (Nianogo et al., 1996). Le poids moyen de la carcasse observé dans les élevages traditionnels de la République Démocratique du Congo est de $35 \mathrm{~kg}$ (FAO, 2012). La viande des porcs engraissés en élevage traditionnel a un $\mathrm{pH}$ ultime faible et une capacité de rétention d'eau élevée (Youssao et al., 2004). Du point de vue environnemental, une ambiance climatique de 26 à $30{ }^{\circ} \mathrm{C}$ est favorable à l'expression du potentiel de croissance du porc local (Nonfon, 2005). Avec des rations optimalisées en énergie et en protéine, Il a été possible de produire un porc local de $25 \mathrm{~kg}$ en 5 mois au lieu de 10 à 18 mois en élevage traditionnel (Nonfon, 2005). De plus, après 75 jours d'engraissement, les porcelets nourris avec un aliment concentré ont présenté un poids de près de $60 \%$ supérieur à celui des porcelets alimentés traditionnellement (Keambou et al., 2010). A titre indicatif, les gains moyens quotidiens du porc local en engraissement obtenus avec des rations optimales sont de $206 \mathrm{~g} / \mathrm{j}$ (Keambou et al., 2010), $200 \mathrm{~g} / \mathrm{j}$ avec des indices de consommation de 3,86 (Codjo, 2003), 119-127 $\mathrm{g} / \mathrm{j}$ (Youssao et al., 2008), $274 \mathrm{~g} / \mathrm{j}$ avec des indices de consommation de 3,19 (Nonfon, 2005). Tous ces gains moyens quotidiens sont très inférieurs à celui obtenu sur des porcs européens (690 à 790 g/j) (Nonfon, 2005) avec des rations ayant presque les mêmes valeurs que celles utilisées par ces différents auteurs. L'amélioration des conditions d'élevage et particulièrement l'alimentation $\mathrm{du}$ porc indigène d'Afrique est favorable à l'expression de son potentiel zootechnique. Malgré l'optimalisation des rations, les performances zootechniques du porc local restent encore faibles par rapport à celles des races améliorées. Face à cette efficacité alimentaire faible et la grande variabilité des vitesses de croissances observée par certains auteurs (16 à $24 \%$ de la naissance à 5 mois) (Nonfon, 2005), il est important d'explorer le potentiel génétique du porc local en vue de sa mise à contribution dans les programmes d'amélioration des performances de cette race.

\section{DIFFERENTES FORMES D'ELEVAGE PORCIN}

En Afrique et particulièrement au Bénin et même partout dans le monde, les porcs sont élevés de différentes manières.

\section{Elevage domestique}

Pratiqué de manière plus ou moins extensive, le but de l'élevage domestique est de constituer une réserve financière pour la famille et de produire un peu de viande de porc pour la consommation familiale, sans avoir à investir beaucoup de temps et d'argent. L'élevage domestique est courant chez les petits paysans pratiquant diverses cultures et élevages. Au Bénin, cette Pratique d'élevage est appelé élevage traditionnel. L'élevage traditionnel porcin est caractéristique du milieu coutumier non islamisé. C'est le porc local qui est élevé. Dans ce système, les éleveurs consentent un minimum d'investissement et d'intervention pour maintenir la rentabilité de leur exploitation. Dans la plupart des cas, les animaux sont laissés en liberté pour compléter leurs rations journalières qui peuvent être apportées par l'éleveur ou bien trouvées sur les tas d'ordures, sur les lieux d'aisance et dans les champs. Une évolution du système est constatée car tous les éleveurs ne laissent plus leurs animaux en divagation permanente (Bossa, 2009). Cette forme d'élevage est répandue à près de $90 \%$ (Ayssiwede, 2004). 


\section{Elevage porcin semi-intensif}

Il se caractérise par le parcage des animaux dans des bâtiments et un meilleur contrôle de leur nutrition et de leur santé. Les objectifs restent ceux de l'élevage domestique, mais les investissements modestes engagés se traduisent par un meilleur rendement. Dans ce système au Bénin, les animaux plus âgés sont mis en enclos. La cabane est en banco ou en briques et est revêtue de tôles ou de pailles. L'enclos est fait soit en banco soit en grillages métalliques. Ce type d'élevage a pour avantage la réduction du coût et le nettoyage rapide de l'enclos, mais comme inconvénients l'entretien coûteux et la réparation des grillages, la surveillance plus difficile des animaux, la nécessité d'une importante surface, le risque d'infestation (Bossa, 2009).

\section{Elevage intensif}

Il s'agit de produire de façon rentable de la viande de porc pour la vente. On élève alors un grand nombre de porcs. Cette sorte d'élevage demande un investissement important en termes de moyens financiers et de temps, et implique un calcul précis des coûts et des bénéfices réalisés. Appelé élevage porcin moderne au Bénin, il est peu développé. Il fait appel aux races importées Large White et Landrace. Il est rencontré dans les structures d'organisation d'éleveurs, dans les centres modernes comme le centre Songhaï et la ferme de Kpinnou (Bossa, 2009).

\section{CONTRAINTES MAJEURES L'ELEVAGE DU PORC AU BENIN Contraintes légales}

Selon l'article 45 de la loi n²018-20 du 23 avril 2019 portant Code pastoral en République du Bénin, 'La divagation des animaux domestiques est interdite sur toute l'étendue du territoire national" (Loi n²01820 du 23 avril 2019 portant Code pastoral en République du Bénin, 2019). Cette loi constitue une contrainte majeure pour la divagation des animaux dans l'élevage porcin mais un avantage pour l'installation des Programmes d'amélioration des potentiels Zootechniques de cette race au sein des ménages à faible revenu.
Contraintes liées à la conduite de l'élevage

La conduite d'élevage traditionnel a pour conséquence la faible productivité des animaux à cause de la mauvaise alimentation, du caractère non contrôlé de l'accouplement. Les éleveurs sont aussi confrontés à l'absence de géniteurs souhaités dans le troupeau (Bossa, 2009). S'agissant des élevages semi-modernes et modernes, les contraintes sont axées sur la faible connaissance des techniques de formulation des aliments concentrés appropriées à l'espèce et à son stade physiologique, la rupture de stock des ingrédients ou des concentrés alimentaires. Le coût des intrants est élevé pour les élevages installés en zones périurbaines. A cela, il faut ajouter la rareté de fourrage en période sèche.

\section{Contraintes liées à l'habitat}

Les contraintes sont souvent liées à l'absence d'abri approprié pour les animaux qui cohabitent généralement avec les autres espèces d'animaux. Les habitats qui existent sont en général précaires (Agbokounou et al., 2016) faits de matériaux locaux.

\section{Contraintes liées à l'alimentation et à l'abreuvement}

Les aliments que reçoivent les animaux, de la part de l'éleveur en général, ne suffisent pas pour couvrir les besoins énergétiques de croissance et de production de l'animal. La plupart du temps, les animaux sont en divagation, à la recherche de nourriture. Ceci nécessite de leur part une dépense importante d'énergie qui aurait dû être mobilisée pour la croissance et la production. Les animaux s'abreuvent le plus souvent dans les flaques d'eaux et les eaux grasses qui trainent dans les maisons. Dans ces conditions d'élevage, le porc est exposé à de nombreuses pathologies (Bossa, 2009).

\section{Contraintes sanitaires et pathologiques}

L'élevage de porc au Bénin est confronté à diverses maladies infectieuses et parasitaires. En 1997, des cas de peste porcine africaine ont été enregistrés sur tout le territoire béninois. Les infestations parasitaires entravent sérieusement la croissance des 
animaux. Les maladies dues aux nématodes digestifs et aux ectoparasites occupent une place importante dans ces infestations (Rouillé et al., 2014). Les maladies sont plus importantes dans les systèmes intensifs parce que, les animaux étant nombreux dans un espace limité, les maladies contagieuses peuvent se transmettre rapidement d'un animal à l'autre. De plus, dans les systèmes intensifs d'élevage porcin, les races locales sont remplacées par les races dites améliorées, qui offrent souvent moins de résistance aux maladies. Fort malheureusement, pour plusieurs de ces maladies, exemple de la peste porcine, il n'existe pas encore de vaccin (Soglohoun \& Youssao, 2017).

\section{PARASITES INTERNES DU PORC AU BENIN : DONNEES SCIENTIFIQUES RAPPORTEES AU BENIN ET DANS LES PAYS EXPORTATEURS DU PORC VERS LE BENIN}

Très peu de travaux ont été menés sur le parasitisme interne du porc local au Bénin. Les chercheurs ayant travaillé sur quelques parasites n'ont point mené des études assez approfondies. En 1983, une étude consacrée aux nématodes des porcins dans le département du Zou a révélé un taux d'infestation de 57,26\% (Dossou-Gbété \& Ayite, 1983). Glodjinon, (1997) a rapporté un taux d'infestation des porcs par les nématodes de $68,59 \%$. Par ailleurs, les travaux menés dans les départements de l'Ouémé et du Mono ont montré un taux global de 55,53\% des porcs infestés par les nématodes (Bossa, 2009).

\section{Ascaris suum}

La parasitose engendrée est l'ascaridiose. Elle est retrouvée chez les porcs porcelets et les porcs d'engraissement. L'espèce retrouvée chez l'homme est Ascaris lumbricoides. Mais l'homme peut être sensible à l'ascaris du porc et vice versa. Ascaris suum, ver blanc de l'intestin grêle est un nématode qui mesure 10 à $30 \mathrm{~cm}$ de long (Dufour, 2015). $\mathrm{Au}$ Bénin, l'ascaridiose avec la strongylose font partie des pathologies dominantes de l'élevage porcin, avec une proportion de près de 65\% (Ayssiwede, 2004). Au Nigéria, l'un des pays exportateurs du porc vers le Bénin, l'incidence parasitaire de Ascaris suum dans les regions de Jos entre janvier 1987 et mars 1988 était de 53,1\% (Salifu et al., 1990).

\section{Stephanurus dentatus}

Stephanurus dentatus est un nématode de la famille des Syngamidés, responsable de la stéphanurose porcine. Le parasite se développe dans le foie puis dans les reins du porc. Les signes cliniques de cette parasitose sont généralement inapparents. Cependant, elle peut entraîner des amaigrissements, des retards de croissance, ainsi qu'une raideur du train postérieur, voire une paralysie (Cauquil et al. 2014). Dans de très rares cas ou lors de fortes infestations, elle peut entraîner la mort. Stephanurus dentatus est sensible à l'ivermectine, au fenbendazole et au lévamisole.

\section{Macracanthorhynchus sp.}

La parasitose engendrée est l'acanthocéphalose. Macracanthorynchus hirudinaceus est parasite du porc et de l'homme. Les œufs de Macracanthorhynchus $s p(90-110 \times 50-65 \mu \mathrm{m})$ sont de forme ovoïde et ont une coque caractérisée par trois enveloppes distinctes dont la plus externe présente une ornementation en réseau caractéristique. A l'état adulte, le parasite se fixe dans les intestins de l'hôte et mesure entre 30 et $35 \mathrm{~cm}$ de long (Dufour, 2015).

\section{Strongyloides ransomi}

Le parasite a pour site la muqueuse de l'intestin grêle et entraîne la strongyloidose, une helminthose. Les jeunes animaux sont les plus sensibles et en particulier les porcelets sous la mère et les animaux sevrés. En Afrique, elle est d'autant plus fréquente que l'on se rapproche de l'équateur. Au Nigeria, par exemple, la prévalence varie de 0 à $87 \%$ selon la localisation des élevages (Bossa, 2009). Au Bénin, la strongyloidose ensemble avec l'ascaridiose fait partie des pathologies dominantes de l'élevage porcin, avec une proportion de près de 65\% (Ayssiwede, 2004). Au Nigéria, pays exportateur du porc vers le Bénin, la strongyloidose a également une forte 
prévalence. Dans la région de Jos au Nigéria, Strongyloides ransomi a été isolé du Porc, avec une incidence parasitaire de $87,7 \%$ (Salifu et al., 1990).

\section{Trichuris suis}

A l'état adulte, le parasite est localisé dans les intestins de l'hôte unique. L'infestation survient par ingestion involontaire d'œufs embryonnés provenant $\mathrm{du}$ sol et présents dans la nourriture ou l'eau de boisson. Après ingestion les œufs embryonnés éclosent dans l'intestin grêle puis les larves migrent jusqu'au cæcum où elles s'attachent à la muqueuse par leur extrémité antérieur (Dufour, 2015). Tout comme l'ascaridiose, la trichuriose ou trichurose, est également liée à un problème d'hygiène corporelle ("péril fécal"), à l'utilisation de matières fécales comme engrais dans les cultures ou à une mauvaise gestion des déchets organiques (voie de passage et sols insuffisamment nettoyés) (Dufour, 2015). Au Nigéria, l'un des pays exportateurs du porc vers le Bénin, l'incidence parasitaire de Trichuris suis dans les régions de Jos entre janvier 1987 et mars 1988 était de $8,5 \%$ (Salifu et al., 1990).

\section{Spirures}

On regroupe sous le terme de "Spirures" les Nématodes Secernentea, de l'ordre des Spirurida appartenant à la super-famille des Spiruroidea, famille des Spiruridés, sousfamille des Ascaropsinés. Chez le porc, on en retrouve trois espèces de Spirures (Dufour, 2015). Il existe également un autre Spirure dont les œufs peuvent être retrouvés en coproscopie porcine : Gongylonema pulchrum. Ces parasites ne provoquent pas de symptômes notables sauf si le niveau de parasitisme est élevé et que les animaux soient affaiblis par d'autres facteurs ou chez les porcelets en croissance. Les vers irritent la muqueuse gastrique et y provoquent une inflammation. On observe une gastrite chronique avec des signes de nausées et vomissement, polydipsie, coloration sombre des selles (méléna), inappétence et on note un retard de croissance chez les jeunes (Santolini, 2004).

\section{Genre Fasciola}

La parasitose engendrée est la Fasciolose. Les œufs du genre Fasciola ou grande douve sont de grande taille (130-145 x 70-90 $\mu \mathrm{m}$ en moyenne), de forme ovoïde avec une ouverture polaire ou opercule. Le cycle des taxons de la classe des Fasciolidae est hétéroxène et fait intervenir un hôte intermédiaire et un hôte définitif. Il passe obligatoirement par le milieu aquatique (Dufour, 2015).

Le cycle de ce parasite est hétéroxène et fait intervenir deux hôtes intermédiaires, un gastéropode terrestre puis une fourmi, et un hôte définitif, un herbivore, principalement le mouton, les bovins, les chèvres (Santolini, 2004). L'herbivore peut être atteint de troubles digestifs, de fièvres, son état général peut s'altérer et il peut y avoir des manifestations neurologiques. L'homme et le porc peuvent être porteurs de cette parasitose (Dufour, 2015).

\section{Taenia solium}

Le parasite entraine une cestodose larvaire due à la présence et au développement dans les muscles striés du porc, de larves vésiculaires de type cysticerque. L'espèce en cause est Cysticercus celllulosae, la larve de Taenia solium de l'homme (Santolini, 2004). Ces infections sont banales dans les zones où les villages n'ont pas d'accès aux latrines et où les porcs sont errants et ont accès aux fèces humains. Les données scientifiques disponibles sur la prévalence de Teania solium chez les porcs au Bénin sont peu abondantes. Des inspections sanitaires réalisées au SudBénin ont abouti à d'importantes saisies de viandes de porc pour cause de contamination au parasite. En effet, entre 2007 et 2010, le Bureau d'inspection de la santé animale du Bénin a saisi 4474 organes de viande de porc pour raison de la cysticercose porcine: cœur, cou les muscles, les muscles psoas, la langue et la tête. Les sites avec la plus grande proportion de saisies d'organes étaient respectivement Dogbo, Klouekanme, Aplahoue et Lokossa (Goussanou et al., 2013) Le taux d'infestation des porcs est bien plus élevé lors d'élevage en extérieur dans les 
zones rurales que lors d'élevage en intérieur.

\section{Genre Hyostrongylus}

La parasitose engendrée est l'Hyostrongylose ou «Syndrome de la Truie mince (Uzal et al., 2016). Les vers appartiennent à l'ordre des Strongylida et à la famille des Trichostrongylidae. Une seule espèce a été décrite. Il s'agit de Hyostrongylus rubidus (Bossa, 2009) qui est un nématode trichostrongylidique au cycle biologique typique. Au Nigéria, l'un des pays exportateurs du porc vers le Bénin, l'incidence parasitaire de Hyostrongylus rubidus dans les regions de Jos entre janvier 1987 et mars 1988 était de 13,1\% (Salifu et al., 1990). Le traitement est basé sur la Doramectin, abamectin, ivermectin, fenbendazole, flubendazole, febantel, oxibendazole, thiophanate, levamisole, et dichlorvos (Uzal et al., 2016).

\section{Genre Oesophagostomum}

Le genre est responsable de la Oesophagostomose chez le porc, une affection associée à la formation de gros nodules intestinaux, plus ou moins fréquentes en régions tropicales; Oesophagostomum quadrispinulatum et Oesophagostomum dentatum sont les principales espèces indexées (Uzal et al., 2016). Il existe peu de données sur la prévalence de ce parasite au Bénin. Par contre, des données interessantes existent au Nigéria et au Burkina-Faso, deux pays qui exportent le Porc vers le Bénin. Par exemple, Il a été rapporté une incidence parasitaire de $35,1 \%$ pour Oesophagostomum dentatum chez le porc entre janvier 1987 et mars 1988 dans la region de Jos au Nigéria (Salifu et al., 1990). Selon une étude réalisée à l'est du Burkina Faso entre novembre 2001 et octobre 2002, Oesophagostomum spp. a une prévalence de $18 \%$; 100-1 000 EPG (œufs fécaux par gramme) (Youssao et al., 2006).

\section{Isospora suis}

La coccidiose intestinale à Isospora suis est une affection digestive fréquente chez les porcelets. Il s'agit d'une pathologie d'élevage à évolution endémique, plus fréquente lors des mois d'été. La coccidiose se manifeste par une diarrhée non hémorragique, pâteuse jaunâtre (« diarrhée mayonnaise ») sur des porcelets le plus souvent âgés de 6 à 7 jours mais également sur des animaux plus âgés (14-21 jours). La morbidité, faible en début d'évolution, peut rester modérée $(30 \%)$ ou s'élever de façon importante pour concerner jusqu'à $90 \%$ des portées (Pommier, 2003).

\section{IMPACT DES PARASITES INTERNES SUR LES PERFORMANCES ZOOTECHNIQUES DU PORC DE RACE LOCALE}

Les parasites internes affectent fortement les paramètres zootechniques au sein des élevages porcins et tout particulièrement celui de la race locale par son mode d'élevage. En effet, ces parasites se retrouvent beaucoup plus chez les porcelets tandis que les adultes ont développé un certain niveau de résistance. Les truies sont des porteurs sains et contaminent les porcelets à la tété (Santolini, 2004). Ainsi, les signes généraux en cas d'infestation sont un mauvais état général avec des animaux affaiblis, maigres, rachitiques, à croissance retardée donc une importante réduction du GMQ. Dans le cas de la Strongyloidose du porcelet par exemple, une cachexie progressive est observée, la diarrhée, l'anémie et la mort subite du porcelet donc, une augmentation de la mortalité naissancesevrage. Dans le Cas de la Cysticercose porcine, la perte de la carcasse est à noter donc un faible rendement au sein de l'élevage. La productivité numérique, la productivité pondérale sont autant de paramètres qui sont affectés par ces parasites.

\section{MOYENS ORDINAIRES UTILISES PAR LES ELEVEURS DU PORC POUR LUTTER CONTRE LES PARASITES INTERNES ET LEURS LIMITES}

Au Bénin, la filière porcine semble être la plus négligée avec un faible suivi sanitaire des cheptels porcins, le manque de formation, d'appui financier et matériel et de subvention aux éleveurs. Les éleveurs font donc généralement recours aux plantes médicinales dont ils ont connaissance pour traiter les animaux. Mais la limite première de cette 
utilisation des connaissances endogènes est la méconnaissance de la dose optimale à utiliser, la durée moyenne du traitement, les informations sur la toxicité de ces plantes ainsi que les associations de plantes à éviter. La mise en place d'une politique visant à contrôler l'utilisation anarchique de ces plantes médicinales par les éleveurs serait un atout.

\section{PRECAUTIONS A PRENDRE POUR AMELIORER LA SANTE DES PORCS LOCAUX \\ Prophylaxie sanitaire}

Une hygiène rigoureuse est fondamentale pour prévenir la transmission des parasites. Le nettoyage régulier des locaux (avec de l'eau chaude sous pression) et la désinfection associée à une qualité irréprochable de l'eau de consommation pourraient aider. Par ailleurs, un lavage et un brossage de la truie avant la mise-bas est recommandée pour éliminer les éléments parasitaires infestants, souillant la mère. Elle permet d'interrompre le cycle parasitaire à un ou plusieurs niveaux (Santolini, 2004).

\section{Gestion de la prophylaxie médicale}

Il faut effectuer des coproscopies avant ou après traitement afin d'évaluer le type de parasitisme. C'est une étape fondamentale pour choisir le principe actif et le rythme d'administration. Le traitement des truies avant l'entrée en maternité et au moment du sevrage permet de lutter contre Strongyloides et Oesophagostomum. S'il existe un risque de strongyloïdose ou si les mères n'ont pas été traitées au sevrage, il est recommandé de traiter les porcelets 3 fois $(J+3, J+6, J+9)$. Pour les anthelminthiques, il faut y faire recours par un choix judicieux basé sur le spectre d'action recherché, l'innocuité des principes actifs, la facilité d'emploi et le coût. Il faut noter que l'utilisation des anthelminthiques en continu dans l'alimentation permet une meilleure activité anthelminthique et ne nécessite pas de manipulation des animaux (Santolini, 2004). Une étude réalisée au Bénin a montré que l'Ivermectine, le Lévamisole, le Tétramisole, le Citrate de pipérazine, l'Albendazole, l'Oxbendazole et l'Oxfendazole sont les produits vétérinaires utilisés contre les parasites internes du porc au Bénin (Ayssiwede, 2004).

\section{PERSPECTIVES POUR AMELIORER LA GESTION DE CES PARASITES CHEZ LES PORCS}

Une politique de gestion des parasites internes du porc de race locale en Afrique doit partir des moyens locaux les plus accessibles et moins chers aux éleveurs par exemple l'accès. aux plantes médicinales, il serait donc judicieux de mener des études approfondies sur les plantes médicinales afin d'identifier les composés bioactifs qu'elles contiennent, étudier leur toxicité et déterminer la dose optimale pour une efficacité exponentielle et une bonne prise en charge des parasites intestinaux.

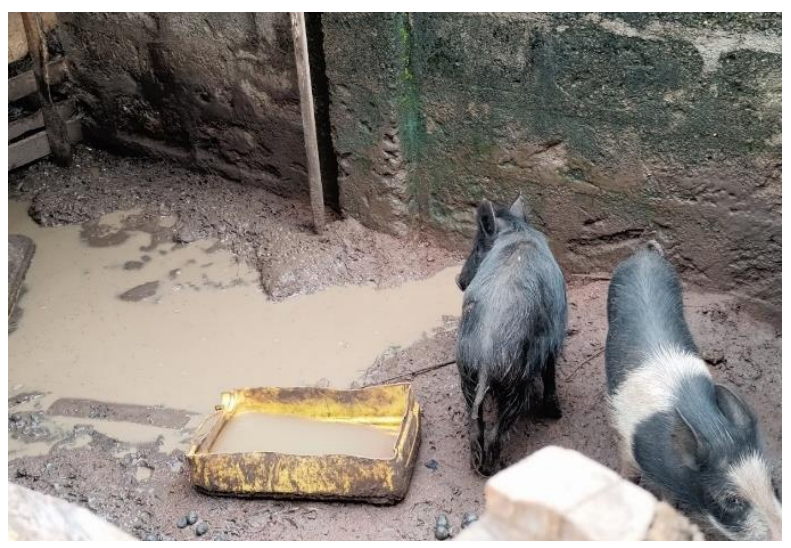

Photo 1 : Enclos en bourbier pendant la saison des pluies (Source : Photo originale). 
A. C. C. ADOHO et al. /Int. J. Biol. Chem. Sci. 15(4): 1698-1716, 2021

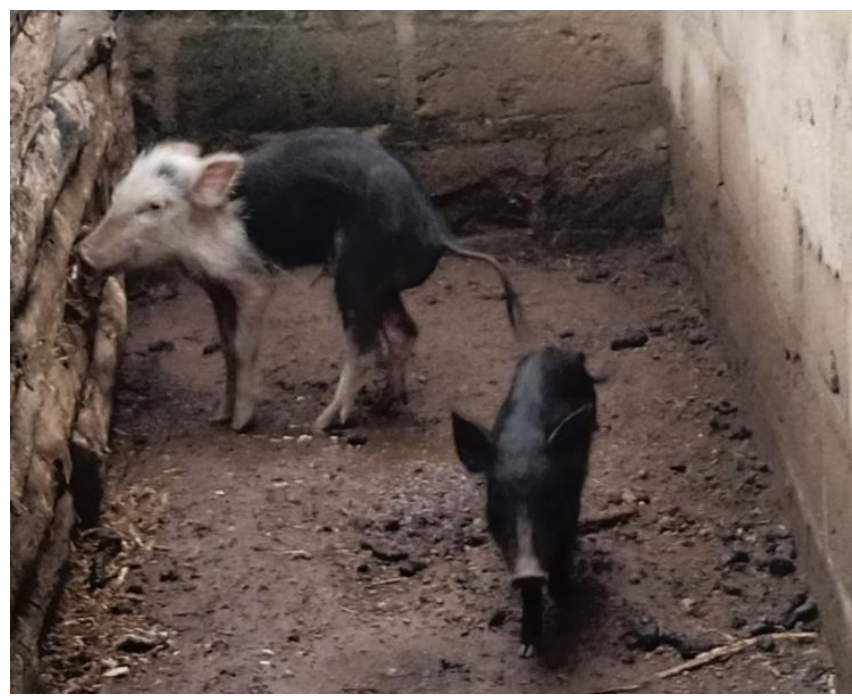

Photo 2 : Porcs locaux adultes (Source : Photo originale).

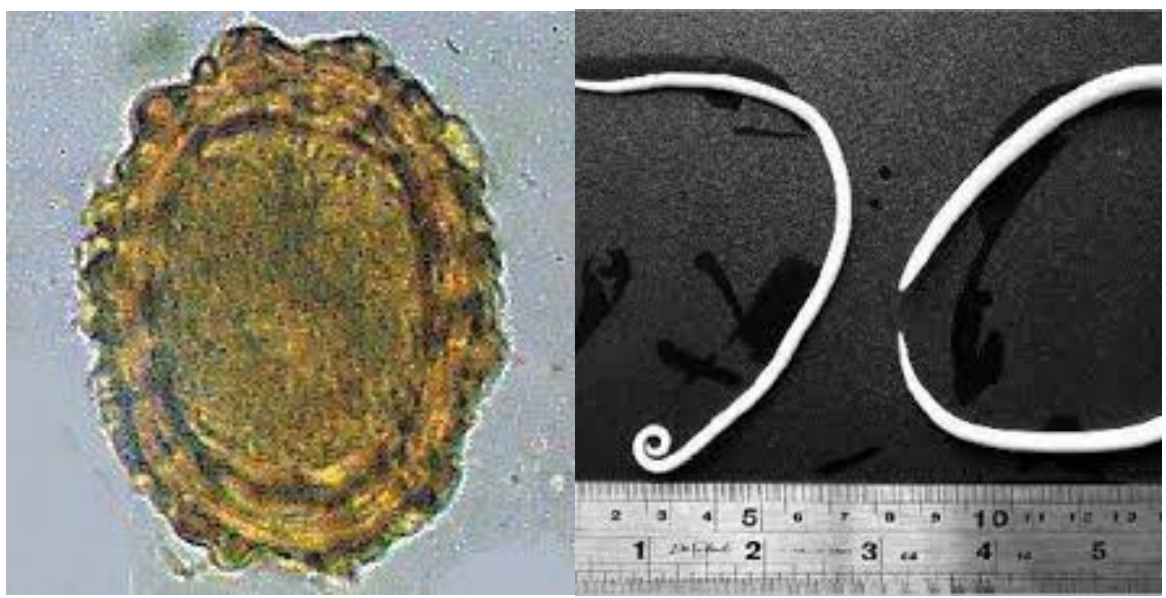

Figure 1 : Euf et vers adultes de Ascaris suum (Alchetron, 2017; Stewart et al., 2021).
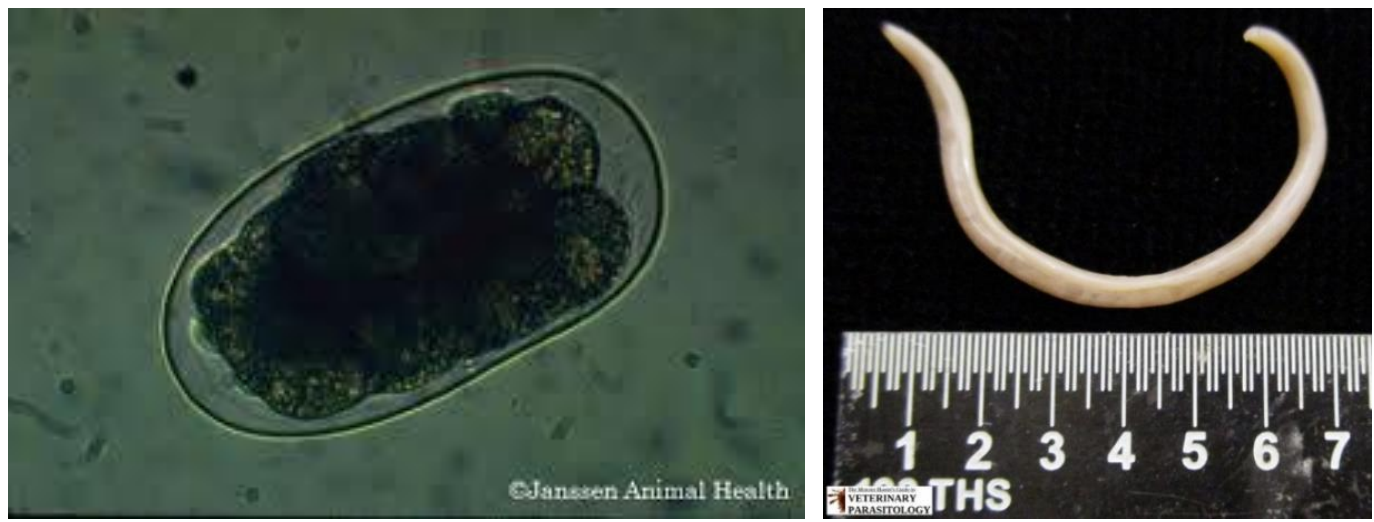

Figure 2 : Eufs et vers adultes de Stephanurus dentatus (Brandy, 2013; Lance, 2019b). 


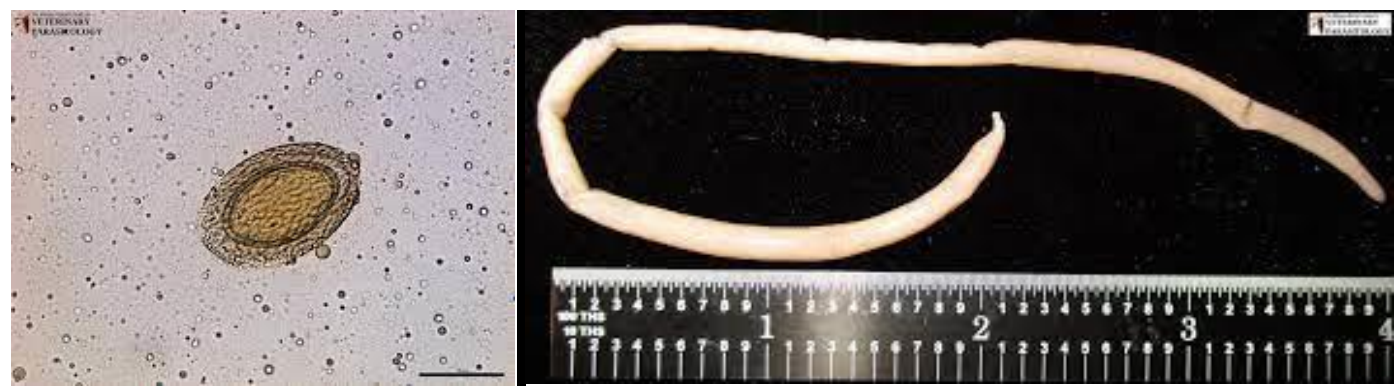

Figure 3 : Eufs et vers adultes de Macracanthorhynchus sp.(Lance, 2019a).
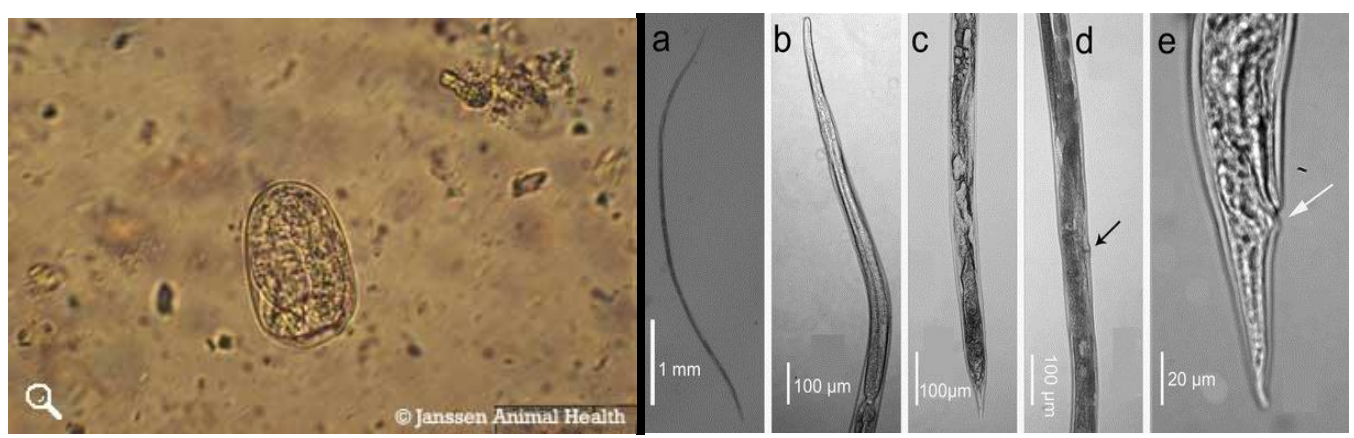

Figure 4 : Eufs et vers adultes de Strongyloides ransomi(The RVC FAO, 2021; Tran, 2018; Viney, 2007).

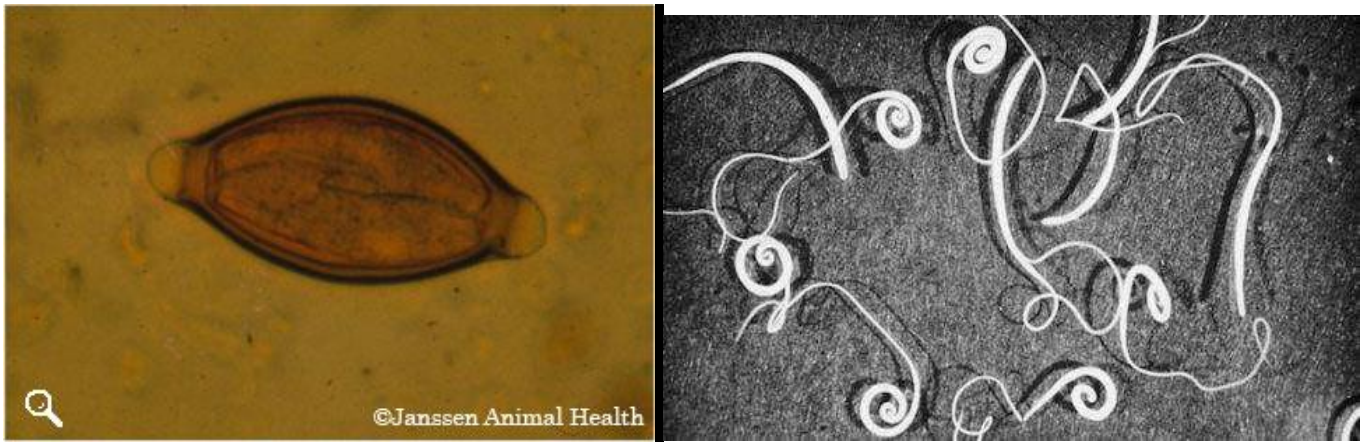

Figure 5 : Eufs et Vers adultes de Trichuris Suis(The RVC FAO, 2021b; Stewart et al., 2021). 

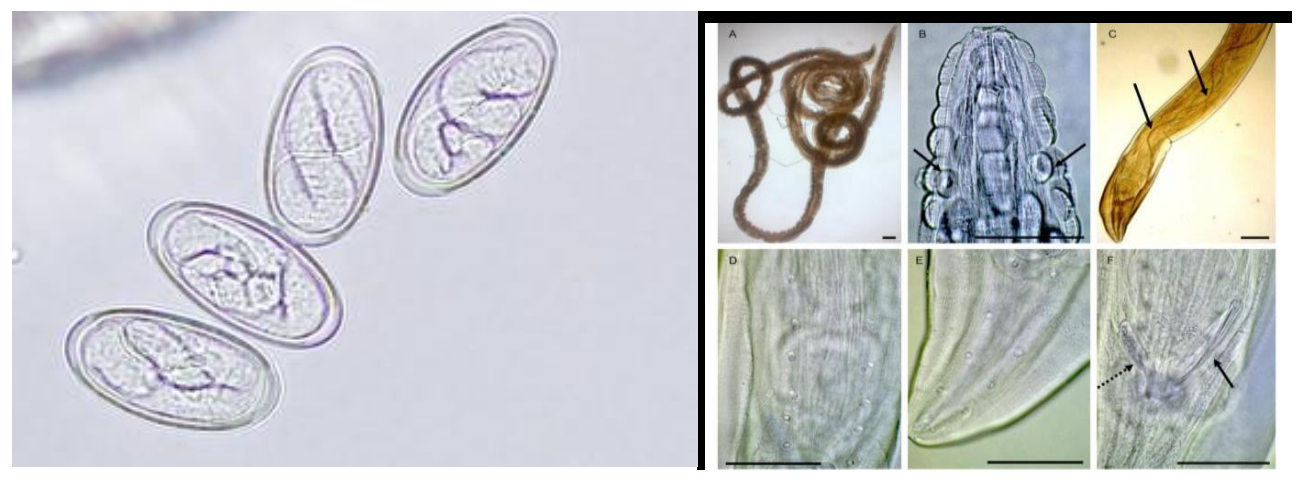

Figure 6 : Eufs et vers de Gongylonema pulchrum. (Ward, 1916).
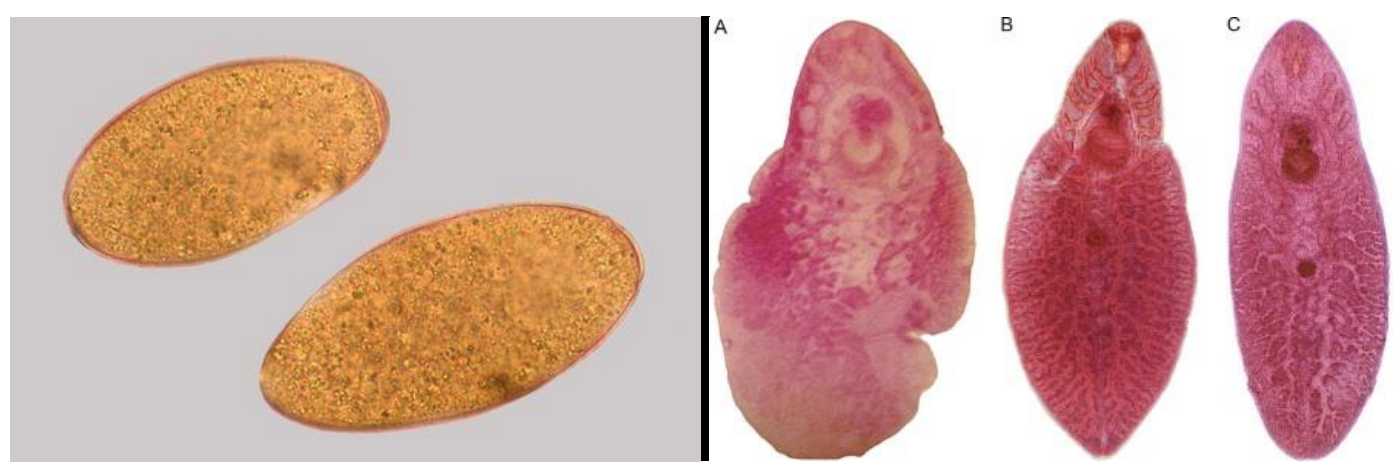

Figure 7 : Fasciola hepatica œuf (à gauche) et mature (à droite) (Hanchante herrera, 2021; Zhou et al., 2008) Genre Dicrocoelium.

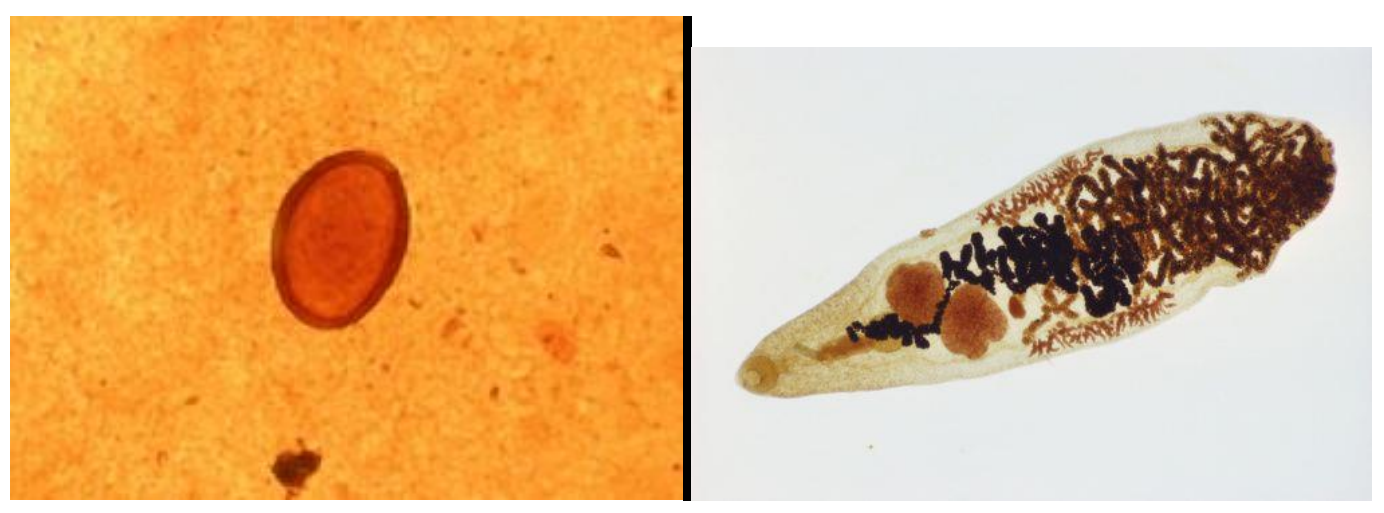

Figure 8 : Dicrocoelium dendriticum œuf (à gauche) et mature (droite) (Molina, 2021; The Merckt Veterinary Manual, 2008). 


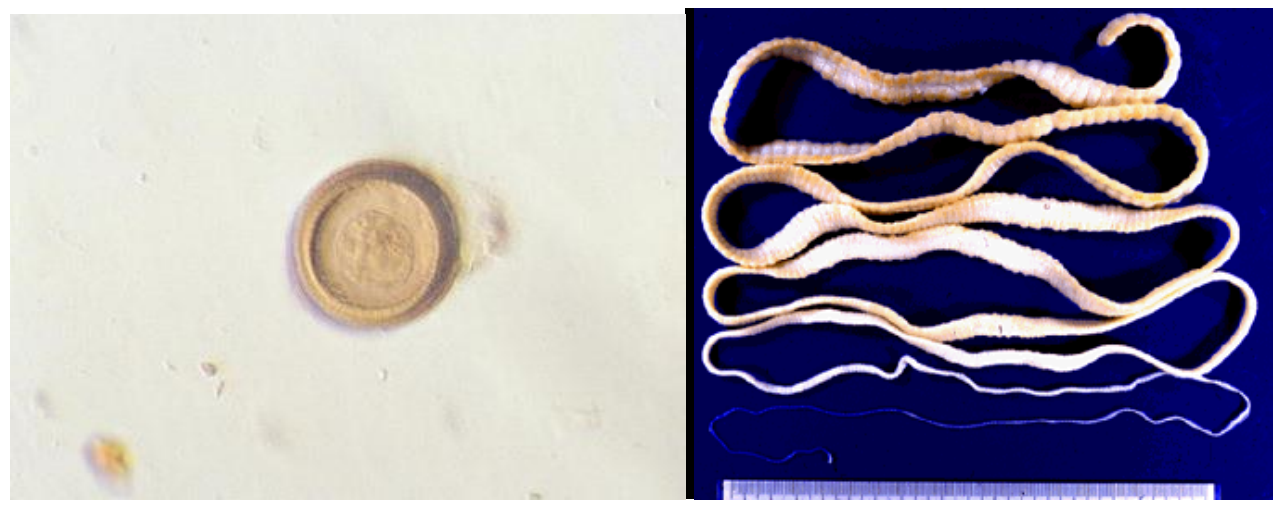

Figure 9 : Eufs et vers adultes de Taenia solium(Brown, 2012; Mehlhorn, 2016).
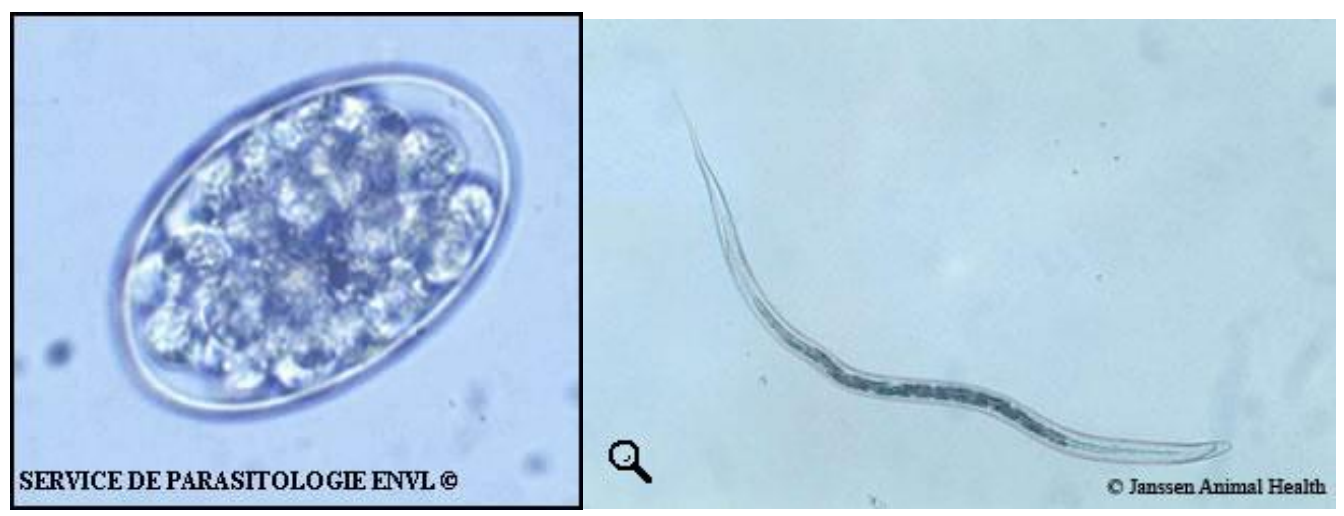

Figure 10 : Eufs et vers adultes de Hyostrongylus rubidus(Alizarine, 2021; The RVC FAO, 2021).
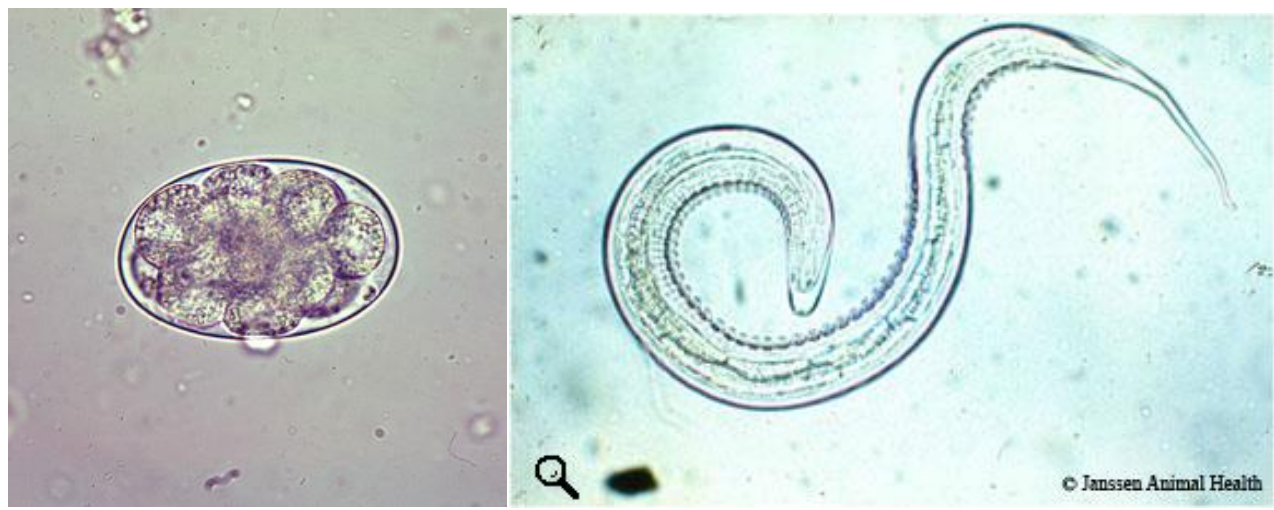

Figure 11 : Eufs et ver adulte de Oesophagostomum dentatum (MCD International, 2021). 


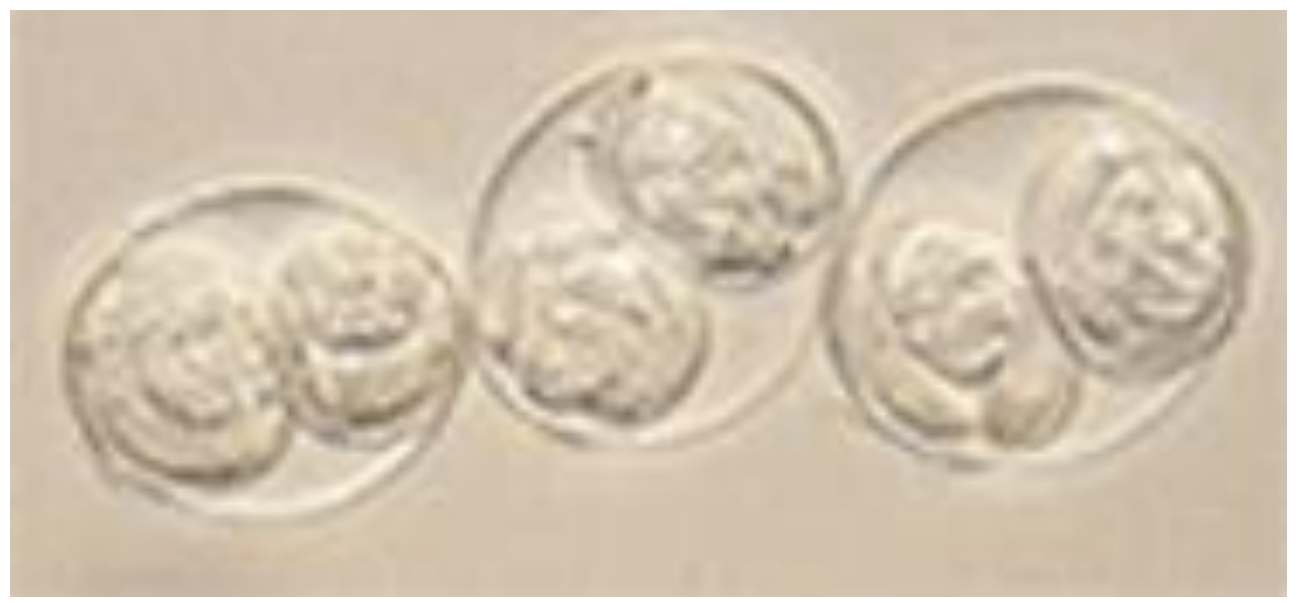

Figure 12 : Oocyste d'Isospora suis (Carrs consulting, 2021).

\section{CONCLUSION}

Cette revue de littérature a montré que malgré son importance au sein de l'économie agricole béninoise et la volonté manifeste des populations paysannes à en faire une activité économique rentable, l'élevage du porc de race locale est confronté à un certain nombre de problèmes dont le parasitisme interne. L'importance médicale des parasitoses tient bien plus à leur fréquence qu'à la gravité des troubles qu'elles occasionnent. Le polyparasitisme est en effet un frein à la rentabilité des élevages porcins. L'absence de données précises sur la prévalence des parasites aggrave la situation. Il urge d'entreprendre des études de nature épidémiologique sur les parasites interne du porc au Bénin afin de mettre en place des stratégies adéquates de leur prise en charge.

\section{CONFLITS D'INTERETS}

Les auteurs déclarent qu'il n'y a pas eu de conflit d'intérêts.

\section{CONTRIBUTIONS DES AUTEURS}

PAO a réalisé la conception de la recherche et a supervisé le travail, ACCA a collecté les données à travers une méthodologie appropriée et a rédigé la première version du manuscrit, EVBA, SH-A et $\mathrm{ABG}$ ont évalué et révisé le manuscrit pour soumission.

\section{REMERCIEMENTS}

Les auteurs remercient le Gouvernement Béninois pour leur appui financier à travers le Programme d'Appui à $\mathrm{M}^{\text {lle }}$ Akouavi Carine Chimène ADOHO, doctorante à l'Ecole Doctorale des Sciences Agronomiques et de l'Eau (EDSAE) de l'Université Nationale d'Agriculture (UNA).

\section{REFERENCES}

Abdallah-Nguertoum E. 1997. Élevage porcin en région périurbaine de Bangui(Centrafrique). Thèse de Docteur Vétérinaire. École Inter - États des Sciences et Médecine Vétérinaires, Dakar, p. 111

Agbokounou AM. 2001. Etude des besoins énergétiques et protéiques du porc local béninois en phase de démarragecroissance Mémoire de DEA en Zootechnie. Faculté Universitaire des Sciences Agronomiques de Gembloux, Gembloux, p. 92

Agbokounou AM, Ahounou GS, Youssao AI, Mensah GA, Koutinhouin B, Hornick JL. 2016. Caractéristiques de l'élevage du porc local d'Afrique. Journal of Animal \& Plant Sciences, 30(1) : 4701- 4713.

Alchetron. 2017. Ascaris suum Detailed Information | Photos | Videos. 
Alchetron.Com. Consulté le 21 Juillet $2021 \mathrm{https} / / /$ alchetron.com/Ascaris-suum Alizarine. 2021. Oeuf_ Hyostrongylus_rubidus. Consulté 21 juillet 2021 , http://alizarine.vetagrosup.fr/coproparasi te/sommaire/diagnostic_par_especes/por cs/page_photo/p_hyostrongylus.htm

Anugwa F \& Okwori A. 2008. Performance of growing pigs of different genetic groups fed varying dietary protein levels. African Journal of Biotechnology, 7: 2665- 2670.

Ayssiwede S. 2004. La filière porcine au Bénin: Production, commercialisation, propositions d'amélioration et perspectives de développement. Thèse Méd. Vêt. Ecole Inter-états des Sciences et Médecine Vétérinaires, Université Cheikh Anta Diop de Dakar, p.161

Stewart BT, Stromberg BE, Bellm A, Biehl L, Oetting S. 2021. Internal Parasites in Swine.

https://www.clemson.edu/extension/4h/p rograms/aganimals/livestock/files/youtha cadmy/swine/module3-biosecurity/pih44-internal-parasites-swine.pdf

Bossa AC. 2009. Nématodes du tube digestif et ectoparasites du porc local dans le SudBénin: Cas des départements de l'Ouémé et du Mono. Thèse pour l'obtention du grade de docteur Vétérinaire. Université Cheick Anta Diop. p.106.

Brandy B. 2013. Stephanurus dentatus Nematode | Nematode, Parasite. Pinterest.

https://www.pinterest.com/pin/40637956 6346002363/

Brown T. 2012. Themes of Parasitology: Taenia solium: Its Eating Your Brain!. http://bio390parasitology.blogspot.com/2 012/03/taenia-solium-its-eating-yourbrain.html

Carrs consulting. 2021. Wormeggcount. http://www.carrsconsulting.com/thepig/b asicvet/wormeggcount/wormeggcount.ht $\mathrm{m}$

Carter N, Dewey C, Mutua F, de Lange C, Grace D. 2013. Average daily gain of local pigs on rural and peri-urban smallholder farms in two districts of
Western Kenya. Tropical Animal Health Production, 45: 1533- 1538.

Cauquil L, Barrière P, Hüe T. 2014. Prévalence de Stephanurus dentatus chez le cochon féral (Sus scrofa) en Nouvelle-Calédonie. Revue d'Elevage et de Médecine Vétérinaire des Pays Tropicaux, 66(4): 131- 135.

Codjo A. 2003. Estimation des besoins énergétiques du porc local du Bénin en croissance entre 7 et $22 \mathrm{~kg}$ de poids vif. Tropicultura, 21 : 56- 60.

Corrégé I, Barbot F, Hémonic A, Pinsard J-L. 2008. Facteurs de risque associés aux niveaux de séroprévalence en salmonelles d'élevages de porcs naisseursengraisseurs et engraisseurs. Journées Rech. Porcine, 40 : 1 - 6.

Direction de l'élevage. 2015. Direction de l'élevage. p. 80 Rapport annuel d'activité. MAEP.

Djimenou D, Adoukonou-Sagbadja H, Koudandé DO, Chrysostome,CAAM, Hounzangbé-Adoté SM, Agbangla C. $2017 . \quad$ Caractéristiques sociodémographiques des éleveurs de porcs (Sus Scrofa domesticus) et structure du cheptel porcin au Sud du Bénin. International Journal of Biological and Chemical Sciences, 11(5): 2177-2193. DOI:

https://dx.doi.org/10.4314/ijbcs.v11i5.19

Do Duc L. 2013. Caractères génétiques et zootechniques du porc Piétrain stress négatif en croisement avec les truies exotiques et locales au Nord du Vietnam. Thèse de Docteur en Sciences Vétérinaires. Faculté de Médecine Vétérinaire, Université de Liège. p.160.

d'Orgeval R. 1997. Le développement de l'élevage porcin en Afrique: L'analyse des systèmes d'élevage du porc local africain au Sud Bénin. Thèse de Doctorat de l'Institut National Agronomique, Paris Grignon, p273.

Dossou-Gbété G, Ayité C. 1983. Contribution à l'étude de quelques nématodes parasites des porcins dans la province du ZOU, Mémoire de fin d'études, Collège Polytechnique Universitaire, Bénin, p 88. 
Dufour B. 2015. Synthèse de données et nouvelle contribution à l'étude des parasites de l'époque romaine, et apports méthodologiques de l'extraction des marqueurs au traitement des résultats. Thèse pour l'obtention du grade de docteur en Archéologie, Territoire et Environnement, Université de Bourgogne, p. 363.

FAO. 2012. Secteur Porcine République Démocratique du Congo. ( $\mathrm{N}^{\circ} 2$ ).

FAOSTAT-FAO. 2020. Equilibres des produits -Elevage et pêche-Equivalent primaire.

www.fao.org/faostat/fr/\#data/QA

Glodjinon JCD. 1997. Les nématodes du tube digestif des porcins dans le Département de l'Ouémé Enquête parasitologique par autopsies helminthologiques, Mémoire de fin de cycle, Collège Polytechnique Universitaire CPU, Bénin, p. 49.

Goussanou SEJ, Kpodekon TM, Saegerman C, Azagoun E, Youssao A, Farougou S, Praet N, Gabriël S, Dorny P, Korsak N. 2013. Spatial distribution and risks factors of porcine cysticercosis in southern Benin based meat inspection records. International Research Journals, 4(8): 188- 196.

Hanchante herrera HA. 2021. Fasciola hepatica. Pinterest. Consulté 21 juillet 2021,

https://www.pinterest.com/pin/61762653 $6372662197 / ? \mathrm{~d}=\mathrm{t} \& \mathrm{mt}=$ signup

Keambou T, Manjeli Y, Hako B, Meutchieye F, Awono J. 2010. Effets comparés d'un aliment concentré et de l'aliment traditionnel des éleveurs sur les performances de croissance et économique des porcelets de race locale au Nord Cameroun. Revue d'Elevage et de Médecine Vétérinaire des Pays Tropicaux, 63 : 77- 82.

Koutinhouin GB, Youssao A, Toleba SS, Kpodekon TM, Ahounou GS, Bonou A, Bessanvi J. 2009. Effet du mode d'élevage sur la prolificité des truies de race locale du Bénin et la viabilité de leurs porcelets, de la naissance au sevrage. International Journal of Biological and Chemical Sciences, 3(4): 819-829. DOI: 10.4314/ijbcs.v3i4.47188

Lance W. 2019a. Macracanthorhynchus sp. Thorny-headed Worms. Monster hunter's guide to: veterinary parasitology. https://www.veterinaryparasitology.com/ macracanthorhynchus.html

Lance W. 2019b. Stephanurus sp. Roundworms. Monster hunter's guide to: veterinary parasitology. https://www.veterinaryparasitology.com/ stephanurus.html

Lombe B, Sumbu W, Tshilenge M, Madimba K, Masumu M. 2015. Prévalence de la cysticercose porcine à Taenia solium (Cysticercus cellulosae) dans la province du Kongo Central, République Démocratique du Congo. Sciences, 4(1): 43- 49.

Lukusa B. 2005. Amélioration de la pratique d'élevage de porcs dans les milieux urbains et périurbains de la ville de Kinshasa. Mémoire du Diplôme d'Études Spécialisées en Gestion des Ressources Animales et Végétales en Milieux Tropicaux.

MCD International. 2021.

Oesophagostomiasis-Microscopy

Findings. Consulté 21 juillet 2021, https://www.mcdinternational.org/trainin gs/malaria/english/DPDx5/HTML/Frame $\mathrm{s} /-$

R/Oesophagostomiasis/body_Oesophago stomiasis_mic1

Mehlhorn H. 2016. Taenia solium. In Encyclopedia of Parasitology, Mehlhorn $\mathrm{H}$ (Ed.). Springer: Berlin Heidelberg; 2614- 2621. DOI : https://doi.org/10.1007/978-3-66243978-4_3093

Missohou A, Niang M, Foucher H, Dieye P. 2001. Les systèmes d'élevage porcin en Basse Casamance (Sénégal). Cahiers Agricultures, 10 : 405408.

Molina GM. 2021. Dicrocoelium dendriticum. Pinterest. Consulté 21 juillet 2021, 
https://www.pinterest.com/pin/32918505 3985430611/

Mopate L, Kabore-Zoungrana C, Facho B. 2011. Des troupeaux et performances des élevages porcins de la zone de N'Djaména au Tchad. International Journal of Biological and Chemical Science, 5(5): 321-330.

Mopate L, Koussou M, Nguertoum E, Ngo Tam, A, Lakouetene T, Awa D, Mal Mal H. 2010. Caractéristiques et performances des élevages porcins urbains et périurbains des savanes d'Afrique centrale : Cas des villes de Garoua, Pala et Bangui. «Savanes africaines en développement : innover pour durer » $\mathrm{du}$ 20-23 avril 2009, Garoua, Cameroun.

Mushandu J, Chimonyo M, Dzama K, Makuza S, Mhlanga F. 2005. Influence of sorghum inclusion level on performance of growing local Mukota, Large White and their F1 crossbred pigs in Zimbabwe. Animal Feed Science and Technology, 122: 321 - 329.

Nianogo A, Sanfo R, Kondombo S, Neya S. 1996. Le point sur les ressources génétiques en matière d'élevage au Burkina Faso. Bulletin d'information sur les ressources génétiques animales, 17 : 11- 28.

Nonfon WR. 2005. La filière de production du porc local au Bénin : L'amélioration de sa productivité par l'alimentation. Thèse de Doctorat en Sciences agronomiques et Ingénierie biologique. Faculté Universitaire des Sciences Agronomiques de Gembloux.

Nonfon, WR, Deka EK, Adegbidji A, Codjo B. 1994. L'élevage du porc local dans le sud Bénin, Enquêtes diagnostiques sur système d'élevage et filière de commercialisation, p. 59.

Ohouko OFH, Koffi K, Dougnon TV, Agbonon A, Messanvi G. 2020. Pratiques d'élevage de porcs au Sud-Bénin : Utilisation des plantes dans la gestion alimentaire et sanitaire. Journal of Animal \& Plant Sciences, 44 : 7677- 7694.

Pommier P. 2003. Systèmes productifs locaux : Les systèmes agroalimentaires localisés : produits, entreprises et dynamiques locales, Montpellier, France, 16 - 18 octobre 2002; CIRAD-TERA. https://agritrop.cirad.fr/514194/

Randriamahefa HT. 2002. Situation de l'élevage porcin dans la commune urbaine et suburbaine de Moramanga, propositions et perspectives d'avenir. Mémoire de fin d'études École Supérieure des Sciences Agronomiques, p. 97

Présidence de la République du Bénin. 2019. Loi n²018-20 du 23 avril 2019 portant Code pastoral en République du Bénin, (testimony of République du Bénin).

Robineau O. 2018. Elever des porcs dans une ville d'Afrique de l'Ouest: Arrangements entre acteurs pour gérer la proximité villeélevage. Revue d'Elevage et de Médecine Vétérinaire des Pays Tropicaux, 71(1-2): 23-31.

DOI: https://doi.org/10.19182/remvt.31288

Rouillé A, Pedrono M, Rakotomalala E, Grosbois V, Ramy-Ratiarison R, Roger F. 2014. Abondance du potamochère, Potamochoerus larvatus, dans les savanes du Nord-Ouest de Madagascar et risques épidémiologiques associés. Bois et Forêts des Tropiques, 320 (320) : 75-82.

Salifu DA, Manga TB, Onyali IO. 1990. Enquete sur les parasites gastrointestinaux chez des porcs du Nigeria (Plateau State et Rivers State). Revue d'Elevage et de Médecine Vétérinaire des Pays Tropicaux, 43(2) : 193-196.

Santolini J. 2004. Le parasitisme interne du porc en zone tropicale. Synthèse bibliographique du DESS-PARC, Cirademvt/Université Montpellier 2, Montpellier France, p.35.

Soglohoun P \& Youssao AKI. 2017. Inventaire des pathologies porcines et mesures de biosécurité dans les départements de l'Ouémé et du Plateau. Mémoire de Licence en Production et Santé Animale, Ecole Polytechnique d'Abomey-Calavi, p. 44.

The Merckt Veterinary Manual. 2008. Dicrocoelium dendriticum. Merck \& Co., Inc. 
http://www.merckvetmanual.com/mvm/i ndex.jsp?cfile $=\mathrm{htm} / \mathrm{bc} / 22705 . \mathrm{htm}$

The RVC FAO. 2021. Pig Eggs: Strongyloides. Consulté 21 juillet 2021, https://www.rvc.ac.uk/review/parasitolog y/pigeggs/Strongyloides.htm

The RVC FAO. 2021. Pig eggs: Trichuris. Consulté 21 juillet 2021, https://www.rvc.ac.uk/review/parasitolog y_spanish/pigEggs/Trichuris.htm

The RVC FAO. 2021. Pig L3 : Hyostrongylus rubidus. Consulté 21 juillet 2021, https://www.rvc.ac.uk/review/parasitolog y/pigl3/hyostrongylus.htm

Tran D. 2018. Morphological and molecular characterisation of Strongyloides ransomi (Nematoda: Strongyloididae) collected from domestic pigs in Bac Giang province, Vietnam. ResearchGate. https://www.researchgate.net/publication 1325891994_Morphological_and_molec ular_characterisation_of_Strongyloides_ ransomi_Nematoda_Strongyloididae_col lected_from_domestic_pigs_in_Bac_Gia ng_province_Vietnam/figures? $1 \mathrm{o}=1$

Umutoni, C. 2012. Évaluation technicoéconomique des élevages de porcs à Bobo-Dioulasso (Burkina Faso). Mémoire de Master en Production Animale et Développement Durable l'École Inter-états des Sciences et Médecine Vétérinaires (EISMV) de Dakar, p. 45.

Uzal F, Prescott J, Songer J, Popoff M. 2016. Clostridial diseases of animals. Wiley Online Library, p. 360.
Viney M. 2007. Strongyloides spp. WormBook. https://doi.org/10.1895/wormbook.1.141. 1

Ward HB. 1916. Gongylonema in the Role of a Human Parasite. The Journal of Parasitology, 2(3): 119-125. DOI: https://doi.org/10.2307/3271194

Youssao AKI, Kpodékon TM, Koutinhouin GB, Adjapka A. 2008. Influence de la castration des mâles sur les performances de croissance, les caractéristiques de la carcasse et les qualités de la viande du porc local du Bénin. Bulletin de la Recherche Agronomique du Bénin, 61 : 1 24.

Youssao A, Mourot J, Gbangboche A, Adehan R, Akoutey A, Edenakpo A. 2004. Influence du regime alimentaire sur les performances de croissance et les caractéristiques de la carcasse du porc de race locale au Bénin. RASPA, 2 : 31- 36.

Youssao I, Maes D, Dembele MA, BangaMboko H, Tamboura HH, Bayala B, Traore A. 2006. Prevalence of common gastrointestinal nematode parasites in scavenging pigs of different ages and sexes in eastern centre province, Burkina Faso. Onderstepoort Journal of Veterinary Research, 73(1): 53- 60.

Zhou L, Luo L, You C, Wang B, Xu J, Lin L, Xuhui H, Bowen C. 2008. Multiple brain hemorrhages and hematomas associated with ectopic fascioliasis in brain and eye. Surgical Neurology, 69(5): 516-521. DOI: https://doi.org/10.1016/j.surneu.20 07.03.018. 